\title{
A METODOLOGIA DE PESQUISA: METASSÍNTESE QUALITATIVA
}

Edvonete Souza de Alencar ${ }^{1}$

Saddo Ag Almouloud ${ }^{2}$

\section{Resumo}

A metassíntese qualitativa é uma das modalidades de pesquisa bibliográfica que executam revisões sistemáticas, sendo importante para a busca e definição de alguns aspectos da área de investigação. Identifica as possíveis melhorias que podem ser efetuadas nas teorias, nas metodologias e nas tendências de investigação, observando ainda quais aspectos necessitam ser abordados e aprofundados. O artigo contém a definição do que é metassíntese qualitativa, mencionada por autores como Vosgerau e Romanowski, Romanowski e Ens, Fiorentini, Maranhão e Manrique e Matheus. Aborda os seus benefícios, evidenciando os aspectos positivos para sua realização, assim como suas limitações. Portanto, apresenta como essa metodologia de pesquisa pode ser realizada, mostrando algumas etapas citadas por Matheus. Propõe ainda reflexões, trazendo como algumas investigações implementaram suas investigações usando essa metodologia de pesquisa.

Palavras-chave: Metodologia; Pesquisa; Metassíntese qualitativa

\section{THE RESEARCH METHODOLOGY: QUALITATIVE META SYNTHESIS}

\begin{abstract}
${ }^{1}$ Doutora em Educação Matemática pela PUC- SP .Professora Adjunta da Universidade Federal da Grande Dourados - UFGD - Faculdade de Educação. E-mail: edvonete.s.alencar@ hotmail.com

${ }^{2}$ Doutor em Mathematiques et Applications pela Universitè de Rennes I em 1992. Coordenador do Curso de Pósgraduação em Educação Matemática pela Pontifícia Universidade Católica de São Paulo. Professor na Pontifícia Universidade Católica de São Paulo e na Fundação Santo André. E-mail: saddoag @ gmail.com
\end{abstract}


Qualitative meta-synthesis is one of the modalities of bibliographic research that carry out systematic reviews, being important for the search and definition of some aspects of the research area. It identifies the possible improvements that can be made in theories, methodologies and research trends, while also looking at which aspects need to be addressed and more explicit. The article presents the definition of what is qualitative metasynthesis mentioned by authors such as Vosgerau and Romanowski, Romanowski and Ens, Fiorentini, Maranhão and Manrique e Matheus. It addresses its benefits by highlighting the positive aspects of its achievement and its limitations. This is how this research methodology can be performed, showing some steps cited by author Matheus. It also proposes reflections bringing as some investigations carried out their investigations using this methodology of research.

Keywords: Methodology; Search; Qualitative meta-synthesis

\title{
LA METODOLOGÍA DE INVESTIGACIÓN: METASÍNTESIS CUALITATIVA
}

\begin{abstract}
Resumen
La metasíntesis cualitativa es una de las modalidades de investigación bibliográfica que realizan revisiones sistemáticas, siendo importante para la búsqueda y definición de algunos aspectos del área de investigación. Identifica las posibles mejoras que pueden realizarse en las teorías, las metodologías y las tendencias de investigación, observando aún qué aspectos necesitan ser abordados y profundizados. El artículo presenta la definición de lo que es metasíntesis cualitativa mencionada por autores como Vosgerau y Romanowski, Romanowski y Ens, Fiorentini, Maranhão y Manrique; y Matheus. Aborda sus beneficios evidenciando los aspectos positivos para su realización,así como sus limitaciones. Así presenta como esta metodología de investigación puede ser realizada, mostrando algunas etapas citadas por la autora Matheus. También refleja como algunas investigaciones realizaron esta metodología de averiguación.
\end{abstract}

Palabras clave: Metodología; Investigación; Metasíntesis 


\section{INTRODUÇÃO}

Este artigo tem por objetivo tecer algumas reflexões sobre a metodologia "metassíntese qualitativa", sua diferenciação entre as demais metodologias que executam revisões sistemáticas de investigações, seus benefícios, limitações e como se pode realizá-la. Além disso, mostra alguns estudos que a utilizaram, como o fizeram e os resultados e benefícios obtidos.

As revisões sistemáticas de pesquisas vêm sendo realizadas nos últimos anos por diferentes áreas de investigação, com o intuito de mapear o que tem sido produzido de conhecimento científico. Essa metodologia possui diferentes modalidades, como estado da arte, estado do conhecimento, metanálise e a metassíntese qualitativa. Neste artigo, apresentamos essa última modalidade de modo detalhado. Por serem investigações qualitativas e de caráter bibliográfico, são consideradas, segundo Lakatos (2011, p. 43), como pesquisas de tipo qualitativo que necessitam da realização de tratamentos científicos com o intuito do conhecimento da realidade.

\section{O QUE É METASSÍNTESE QUALITATIVA?}

A metassíntese qualitativa é uma das modalidades de pesquisa que realiza uma revisão sistemática de investigações qualitativas, diferentemente de outras, como o estado da arte, o estado do conhecimento e a metanálise. Vosgerau e Romanowski (2014) mencionam que essas modalidades se dividem em: a) revisões que mapeiam (os estados da arte e os estados do conhecimento) e b) revisões que avaliam e sintetizam (a metanálise e a metassíntese qualitativa).

Assim, o estado da arte, de acordo com Fiorentini (2011), apresenta uma investigação em uma área de estudo que envolve normalmente um grande número de trabalhos e possui como objetivo descrever as tendências de pesquisa em um determinado campo de conhecimento. Romanowski e Ens (2006) afirmam que:

Os estudos realizados a partir de uma sistematização de dados denominada "estado da arte" recebem esta denominação quando abrangem toda uma área do conhecimento nos diferentes aspectos que geraram produções. Por exemplo: para realizar um "estado da arte" sobre "Formação de Professores no Brasil", não basta apenas estudar os resumos de dissertações e teses; são necessários estudos sobre as produções em congressos na área, estudos sobre as publicações em periódicos da área. O estudo que 
aborda apenas um setor das publicações sobre o tema estudado vem sendo denominado de "estado do conhecimento." (ROMANOWSKI; ENS, 2006, p. 39-40).

Com a menção de Romanowski e Ens (2006), observamos que um estado do conhecimento delimita sua busca por investigações de uma área e/ou setor de publicações. Ele abrange, assim, um número menor de dados a serem investigados. Por sua vez, a metanálise qualitativa, para Fiorentini (2011), é uma revisão sistemática de um número reduzido de pesquisas qualitativas. Caracteriza-se pela realização de análises mais profundas, com resultados mais amplos e gerais obtidos por integrações, cruzamentos e contrastes dos dados.

As autoras Maranhão e Manrique (2014) apresentam a diferenciação entre as modalidades metodológicas de investigação. Elas mencionam que "os estados da arte e os estados do conhecimento requerem a coleta de muitos estudos, o que não acontece com a metanálise qualitativa. Em lugar de partir de amplitude, ela parte de poucos estudos para buscar ampliação, generalização" (p. 428). Do mesmo modo, diferenciam a metanálise da metassíntese qualitativa, esta também realizada por meio de poucos estudos, na qual a seleção das pesquisas segue critério pessoal do pesquisador e utiliza interpretações das investigações. A seguir, apresentamos as considerações sobre a metassíntese qualitativa na abordagem de outros autores.

A metassíntese qualitativa é uma metodologia oriunda de pesquisas internacionais, sendo mais utilizada na área da saúde. Os estudos nas demais áreas surgiram especificamente em meados de 1998, pela Fundação Cochrane, que estabeleceu o "Qualitative Research Methods Working Group", que dá orientações metodológicas a pesquisadores que utilizam as revisões sistemáticas de acordo com os padrões definidos pela instituição. Para isso, disponibilizam cursos on-line, apostilas e centros de cooperação internacional, como o "Sandbar Digital Library Project American”, criado para tal fim.

Vosgerau e Romanowski (2014) mencionam que no final do ano 2000 surgiu a Campbell Collaboration, que atua auxiliando pesquisas nas áreas da justiça, da educação, do desenvolvimento internacional, bem-estar social e também de grupos de pesquisadores.

Matheus (2009) define a metassíntese qualitativa como a "integração interpretativa de achados qualitativos (derivados de estudos fenomenológicos etnográficos, da teoria fundamentada dos dados e outros), que são a síntese interpretativa dos dados” (p. 544). Afirma que as integrações mencionadas produzem uma nova interpretação dos resultados e ressalta que esta não pode ser encontrada nos estudos primários analisados. Assim, os estudos primários tornam-se os dados de uma amostra como o todo. A autora acrescenta que a metassíntese 
qualitativa "não é uma revisão resumida com características desvinculadas daquele evento ou daquela experiência, mas sim integra os dados a fim de alcançar um nível teórico mais elevado" (MATHEUS, 2009, p. 544).

A metassíntese qualitativa permite, portanto, que as investigações primárias sejam estudadas com profundidade. As análises podem ser desenvolvidas por meio de relações entre as pesquisas, como comparações, semelhanças e diferenças. Com a análise dos dados e a reflexão sobre eles, surge a metassíntese qualitativa, que apresenta o objeto de investigação com nível elevado de abstração e compreensão.

Matheus (2009) salienta ainda que, para se realizar uma metassíntese qualitativa, é preciso apresentar todos os significados das investigações, mas sem criticá-los ou compará-los por sua eficácia, preservando assim a conduta ética do pesquisador.

Fiorentini (2013) nos diz que a metassíntese qualitativa:

[...] visa produzir interpretações ampliadas de resultados ou achados de estudos qualitativos obtidos por estudos primários (como são as dissertações, teses e pesquisas de professores), os quais são selecionados atendendo a um interesse específico do pesquisador acerca de um fenômeno a ser investigado e/ ou teorizado. (FIORENTINI, 2013, p. 78).

De acordo com o referido autor, a metassíntese qualitativa utiliza interpretações das dissertações e teses analisadas para que o pesquisador as reinterprete, o que Fiorentini (2013) denomina metainterpretação, produzindo assim uma nova explicação e compreensão do tema investigado.

Em consonância com o que é mencionado no estudo de Fiorentini, Vosgerau e Romanowski (2014) observam que essa metodologia de pesquisa permite analisar estudos de ordem qualitativa, conquanto seu objetivo é metassintetizar as conclusões retiradas da análise realizada. Ressalta ainda que, para uma visão interpretativa, é preciso que os resultados encontrados nas análises sejam agrupados e reagrupados por semelhanças, na tentativa de responder à questão norteadora da pesquisa.

\section{OS BENEFÍCIOS E LIMITAÇÕES DA METASSÍNTESE QUALITATIVA}

Segundo Matheus (2009), a função da metassíntese qualitativa é fortalecer os objetivos das pesquisas, visando à melhoria de seus resultados e ao aprimoramento do conhecimento qualitativo, com o intuito de fundamentar a teoria, a prática e os aspectos políticos que 
envolvem as investigações. A autora menciona que essa modalidade de investigação requer uma sensibilidade teórica, pois o pesquisador deverá analisar as pesquisas, descontruindo e construindo reflexões por meio da interpretação e da indução. Assim, esse processo investigativo permite que a pesquisa seja validada por conter aspectos desafiadores e complexos ao pesquisador.

Para Vosgerau e Romanowski (2014), os estudos que utilizam a metassíntese qualitativa promovem a apresentação das contribuições das pesquisas, nas quais podem ser definidos alguns aspectos da área e do campo das disciplinas de investigação. Permitem ainda uma avaliação do que foi produzido academicamente na área, identificando as possíveis melhorias que podem ser realizadas nas teorias e metodologias utilizadas e mesmo se as tendências de investigação ainda necessitam ser abordadas e quais os pontos frágeis que precisam ser aprofundados. Assim, essas investigações são organizadas, esclarecidas e resumem as principais ideias e obras do tema investigado, fornecendo dados existentes na literatura relevante de uma área.

As limitações para a aplicação desse tipo de metodologia iniciam com a busca e minuciosa seleção das pesquisas para a realização da metassíntese qualitativa. Os pesquisadores devem estar cientes de que essa forma de investigação não abarca todas as pesquisas efetuadas, pois essa espécie de estudo é sempre inconclusa porque o período da busca é finito e a coleta depende do que os autores declararam como foco da pesquisa, das palavras-chave, do título e do resumo.

Além disso, após a seleção das investigações, os pesquisadores devem ter acesso ao arquivo completo das dissertações e teses. Porém, algumas vezes, em casos de investigações mais antigas, não estão disponíveis em bancos de dados on-line, o que dificulta a sua disponibilização, visto que se encontram em bibliotecas físicas dos programas de pósgraduação e o seu acesso deve ser realizado utilizando o sistema Programa de Comutação Bibliográfica (Comut). Os estudos de Romanowski e Ens (2006, p. 47) já referiam, como limitação em metodologias de revisões sistemáticas do tipo estado da arte, um "processo dispendioso e demorado do Comut".

Destarte, o uso dessa metodologia traz desafios ao investigador, tanto de ordem prática, com a busca, seleção das pesquisas e seu tratamento de dados, como o processo complexo de realização da metassíntese qualitativa, ao comparar os dados e tecer novas conclusões com o intuito de responder à questão norteadora da investigação. 


\section{COMO DESENVOLVER ESSE TIPO DE PESQUISA?}

Matheus (2009) apresenta algumas etapas da metassíntese qualitativa, indicando que existem dois modos de realizá-la: 1) Pela integração ou agregação dos dados apresentados nas investigações; 2) Pela interpretação das evidências qualitativas.

De acordo com Matheus (2009), nesses dois modos, as etapas para a realização de uma metassíntese qualitativa são:

a) A identificação do interesse do pesquisador pelo tema de estudo e do objetivo da pesquisa. Nessa metodologia é importante estabelecer o objetivo para que se possam buscar e selecionar as pesquisas sobre o tema de investigação de modo adequado. $\mathrm{O}$ objetivo deve ser bem específico, pois norteará a pesquisa. $\mathrm{O}$ interesse pelo estudo, por sua vez, deve estar centrado em sua relevância e na necessidade acadêmica, como, por exemplo, reunir dados dispersos sobre determinado tema;

b) Seleção do que é relevante aos interesses da pesquisa, tendo como norte o seu objetivo. Realiza-se, nessa etapa, a coleta de dados, que deve obedecer a processos criteriosos para que se apresente a validade da metassíntese qualitativa. Para isso, devem-se selecionar criteriosamente as pesquisas relevantes à investigação e realizar uma análise de cada uma delas, utilizando-se para isso os objetivos inerentes à investigação;

c) Realização da leitura e da releitura das investigações com o intuito de identificar as interpretações relevantes para a pesquisa. Devem-se ainda elaborar resumos e fichamentos a fim de deixar os resultados mais claros, acessíveis e organizados;

d) Apresentação de como as investigações estão relacionadas, evidenciando os resultados das pesquisas analisadas. Nessa etapa, são feitas comparações iniciais sobre semelhanças, diferenças e complementaridades entre os estudos;

e) Realização de afirmações mais amplas e fundamentadas que apresentem os resultados das investigações analisadas com a preservação do contexto oriundo da pesquisa;

f) Elaboração de uma nova interpretação em que estejam presentes todos os estudos analisados e responda ao objetivo inicial.

Salientamos que as etapas aqui apresentadas por Matheus (2009) mostram um dos possíveis modos de se realizar a metassíntese qualitativa. 


\section{ALGUMAS PESQUISAS QUE ADOTARAM ESSA METODOLOGIA}

Com o intuito de melhor compreender como esses estudos são efetuados, apresentamos os seus benefícios e limitações e mostramos algumas investigações desenvolvidas na área de Educação Matemática. Descobrimos que ainda há poucos estudos que utilizaram a metassíntese qualitativa, dado que nossa busca no portal de periódicos da Capes resultou em uma tese de Alencar (2016) relacionada à Educação Matemática. Realizamos posteriormente nossa busca no Google acadêmico, no qual encontramos três artigos científicos, um do grupo de pesquisa de Sábado, que é um grupo colaborativo que reúne professores que ensinam Matemática na escola básica, futuros professores e professores/pesquisadores universitários da Unicamp, de autoria de Fiorentini (2013), e dois originários do grupo de pesquisa Professor de Matemática: Formação, Profissão, Saberes e Trabalho Docente, da PUC-SP, Maranhão e Manrique (2014) e Tinti e Manrique (2016).

Como realizamos uma metassíntese qualitativa, uma das modalidades de revisão de pesquisas e documentos, a pesquisa deve apresentar as origens (instituições e regiões em que foram publicadas) e os fichamentos. Neste artigo utilizaremos dois modelos de fichamento: um mais resumido e usado por Neves (2008), que contém: referência completa, referencial teórico, objetivos, métodos e resultados; e o modelo de fichamento de Martins (2008), que possui: título da dissertação, autor, instituição, ano de defesa, número de páginas, palavras-chave, resumo, objetivo, referencial teórico, análises e referenciais bibliográficos.

As dissertações e teses levantadas e selecionadas no Portal da Capes, conforme o objetivo deste trabalho, são os estudos primários aqui considerados - o foco da metassíntese qualitativa, que investigará o uso dos referenciais teóricos que sustentam as pesquisas selecionadas. Assim, começaremos nossa investigação com um grupo de pesquisas, em que buscaremos os referenciais teóricos que as sustentam, e refinaremos a investigação para um grupo menor de pesquisas que utilizam os mesmos referenciais teóricos.

Ressaltamos que essa metodologia pode ser realizada com um número reduzido de pesquisas, podendo ser um estudo profundo visando obter "resultados integrativos, cruzados ou contrastados com o intuito de produzir resultados mais amplos" (FIORENTINI, 2013, p. 78).

Logo, por metassíntese qualitativa entendemos uma nova produção, que não se reduz ao resumo, à descrição ou ao relato do que se encontra nas dissertações e teses selecionadas, como se poderia pensar, mas exibe um aprofundamento no estudo das interpretações das pesquisas 
sobre como estas utilizaram os referenciais teóricos que as sustentam. A metassíntese pretendida nesta investigação é do tipo comparativo, devendo abarcar semelhanças, diferenças e complementaridades teóricas, deflagrando-as.

Alencar (2016), na sua tese, realizou a metassíntese qualitativa de dissertações e teses selecionadas no Banco de Teses da Capes. Para isso, utilizou na sua busca as expressões "Formação de Professores" e "Campo Conceitual Multiplicativo" em títulos, resumos ou palavras-chave, nos programas de Educação e Educação Matemática, com área de concentração em Educação Matemática, o que resultou em 329 dissertações e teses. Por meio dos resumos, a autora selecionou as investigações que mencionavam "Formação Contínua e Anos Iniciais" e encontrou 27 pesquisas. Com as pesquisas, identificou suas origens (instituições e regiões em que foram publicadas) para determinar as regiões brasileiras que elas contemplam.

As etapas empreendidas para o desenvolvimento da pesquisa foram: (i) seleção de dissertações e teses nacionais para buscar os referenciais teóricos que sustentam as pesquisas; (ii) seleção de referências teóricas sobre Formação Contínua de professores dos anos iniciais e Campo Conceitual Multiplicativo. Realizou-se uma apresentação da seleção criteriosa das pesquisas e das referências teóricas que sustentam as dissertações e teses com justificativas de todas as decisões; identificou-se o grupo de dissertações e teses selecionadas e como foi feita a busca pelos referenciais teóricos mais citados e/ou mencionados nas investigações; iii) realização da metassíntese qualitativa dos referenciais teóricos que sustentam um grupo de pesquisas acerca da formação contínua de professores; formaram-se categorias criadas com a identificação das principais semelhanças, diferenças e complementaridades encontradas à luz dos referenciais teóricos que sustentam as pesquisas.

Alencar (2016), ao realizar a metassíntese qualitativa, buscou selecionar as pesquisas que utilizaram as teorias desenvolvidas por Vergnaud e Shulman, que aparecem com maior frequência. Ela identificou nove pesquisas: Santos (2005), Campos (2007), Garcia Silva (2007), Rocha (2011), Alencar (2012), Merlini (2012), Santos (2012), Oliveira (2014) e Junior Silva (2015).

Na realização da metassíntese, a referida autora encontrou seis categorias para o uso do referencial teórico de Shulman: I - focaliza os três primeiros conhecimentos (específico do conteúdo, pedagógico e curricular), definindo-os; II - denomina os sete conhecimentos estabelecidos por Shulman, mas não os define; III - ressalta a importância do conhecimento específico do conteúdo; IV - complementa as ideias de Shulman com relação ao conhecimento pedagógico do conteúdo, contribuindo para a formação profissional docente; $\mathrm{V}$ - considera as 
ideias de Shulman concernentes ao conhecimento específico do conteúdo, contribuindo para a formação dos docentes em Matemática; VI - traz à luz aspectos pouco abordados da teoria nos estudos de Shulman.

Para a realização da metassíntese do uso do referencial teórico de Vergnaud Alencar (2016) encontrou oito categorias: I - apresenta a definição da Teoria dos Campos Conceituais; II - considera um campo conceitual como um conjunto de situações propostas aos estudantes; III - interpreta a noção de esquema na Teoria dos Campos Conceituais; IV - considera o esquema como organização invariante em uma classe de situações; V - apresenta a formação de conceitos pela terna de conjuntos: S - Situações; I - Invariantes; e R - Representações simbólicas; VI - dá grande atenção à construção de conceitos matemáticos; VII - detalha a apresentação do Campo Conceitual Multiplicativo; VIII - traz noções sobre o Campo Conceitual Aditivo (cálculo relacional e cálculo numérico) para o Campo Conceitual Multiplicativo.

Alencar (2016) obteve como resultado primeiramente as contribuições metodológicas, visto que a pesquisa mostrou como se pode realizar uma metassíntese qualitativa e como sua utilização pode ser benéfica em investigações que pretendem realizar revisões sistemáticas. Pode ainda mapear quais pesquisas efetuaram investigações sobre a formação de professores nos anos iniciais sobre o campo conceitual multiplicativo de 1997 a 2015, identificando os referenciais teóricos mais utilizados.

No artigo de Fiorentini (2013), com dados oriundos do Grupo de Sábado, da Universidade Estadual de Campinas (Unicamp), que vem há 17 anos desenvolvendo revisões sistemáticas de investigações. Entre os vários estudos, selecionamos um artigo de Fiorentini (2013) que mostra as investigações que têm sido realizadas no grupo. Apresenta ainda como as investigações vêm se constituindo no grupo de pesquisa e quais estudos realizaram a metassíntese qualitativa.

Fiorentini (2013), em seu artigo "Investigação em Educação Matemática desde a perspectiva acadêmica e profissional: desafios e possibilidades de aproximação", teve o objetivo de problematizar algumas concepções e práticas docentes, análises e discussões sobre a perspectiva acadêmica, apresentando e descrevendo algumas práticas emergentes de pesquisas (FIORENTINI, 2013, p. 61). Esse artigo foi apresentado em uma Conferência no $13^{\circ}$ CIAEM, ocorrida em Recife, no ano de 2011, e republicado nos Cuadernos de Investigación y Formación en Educación Matemática, em 2013. 
Fiorentini (2013) apresenta as concepções de Cochran-Smith e Lytle (1999) para a prática, da prática e na prática. Utiliza o conceito de comunidade de prática de Lave e Wenger (1991), que consideram que há dois tipos de comunidades: a acadêmica científica, constituída por formadores de professores e pesquisadores da universidade, e a comunidade profissional, constituída por professores, formadores de professores e produtores de material didático. Fiorentini apresenta essa base teórica porque desenvolveu em seu grupo de pesquisa estudos que adotaram a comunidade de prática.

O Grupo de Sábado trabalha comumente com quatro tipos de investigação: em diários e por meio de narrativas dos professores; de etapas de pesquisa oral colaborativa; em aulas e ensaios de professores. $\mathrm{O}$ grupo ainda apresenta dois interesses investigativos: a pesquisa dos processos e conteúdos do desenvolvimento profissional docente e a pesquisa dos processos de conhecimentos produzidos pelos professores, seu papel e sua contribuição na colaboração. Esses estudos são realizados por meio de revisões sistemáticas do tipo estado da arte, estado do conhecimento e metanálise; nos últimos anos vêm se realizando também por meio da metassíntese qualitativa.

Fiorentini (2013) menciona que, entre os diversos estudos desenvolvidos pelo grupo, estão especialmente os artigos de Fiorentini de 2009 e de 2010, e também de Fiorentini et al., 2011, que investigam os grupos colaborativos, metassintetizando as práticas reflexivas e narrativas dos professores. Nesses artigos, percebe-se que o autor seleciona um grupo de práticas reflexivas e narrativas dos professores com o objetivo de aprofundar o conhecimento sobre um determinado tema. Salientamos que, nos últimos anos, Fiorentini tem publicado uma variedade de artigos em que pode ser identificada a utilização da metassíntese qualitativa.

Nos artigos apresentados pelo grupo da PUC-SP, Maranhão e Manrique (2014) desenvolveram um estudo intitulado "Pesquisas que articulam a Teoria das Situações Didáticas em Matemática com outras teorias: concepções sobre aprendizagem do professor”, publicado no periódico Perspectivas da Educação Matemática, da Universidade Federal do Mato Grosso do Sul (UFMS). Esse estudo, além de ter como objetivo "investigar articulações entre a teoria das situações didáticas em Matemática e outras teorias, desvelou concepções sobre a aprendizagem do professor com base nos estudos de Cochran-Smith e Lytle" (MARANHÃO; MANRIQUE, 2014, p. 424).

As autoras fizeram uma apresentação dos aspectos históricos da Teoria das Situações Didáticas. Para a realização do estudo, buscaram pesquisas no portal de periódicos da Capes e 
no Google acadêmico, por meio das palavras-chave "teoria das situações didáticas" e "formação de professores", e encontraram cinco artigos.

As autoras leram, refletiram, selecionaram trechos dos estudos e decidiram considerar apenas três artigos, pois eles abordavam adequadamente a Teoria das Situações Didáticas da Matemática e as concepções de aprendizagem do professor. Para isso, elas apresentaram os estudos de Cochran-Smith e Lytle (1999) e suas concepções de conhecimento para a prática, nas quais o professor assume o papel central, desenvolvendo conhecimentos teóricos matemáticos a fim de exercer suas funções. Já o conhecimento na prática é o conhecimento que está nas ações docentes, nas reflexões, nos questionamentos e nas narrativas, que considera os conhecimentos criados pelos docentes, em que a sala de aula se torna cenário de investigação. Os artigos selecionados por Maranhão e Manrique (2014) foram: Oliveira e Scherer (2013); Gontijo, Silva e Carvalho (2012) e Brousseau (2006).

As autoras realizaram as metassínteses observando, em cada artigo, o que foi abordado da Teoria das Situações Didáticas em Matemática em vista das concepções dos conhecimentos docentes.

No artigo de Oliveira e Scherer (2013) foi analisado o papel de um professor formador em uma formação continuada com um grupo de professores do $6^{\circ}$ ao $9^{\circ}$ ano do Ensino Fundamental em Matemática, que discutia o uso de laptops educacionais por meio do software klogo. Esse artigo adotou as ideias construtivistas de Paper e da Teoria das Situações Didáticas de Brosseau. As autoras consideram que esse artigo abordou o conhecimento para a prática, na medida em que observou os conhecimentos teóricos desenvolvidos em uma formação continuada.

O segundo artigo analisado, de Gontijo, Silva e Carvalho (2012), apresenta a informação de sistemas segundo o autor Csikszentmihalyi (1990), para compreender fatores contextuais e a Teoria das Situações Didáticas. Esse artigo mostra a teoria da criatividade como um processo resultante de três sistemas: o indivíduo, o campo e o domínio, considerando o indivíduo como o aluno, o campo como o professor e o domínio, a matemática escolar. Os autores descrevem ainda como foi utilizada a Teoria das Situações Didáticas da Matemática, apresentando quatro fases diferentes: a ação, a formulação, a validação e a institucionalização. Maranhão e Manrique (2014) acreditam que esse artigo se refere ao conhecimento na prática, pois demonstra a necessidade da formação contínua do professor para a elaboração de situações criativas. 
O terceiro artigo metassintetizado é de Brosseau (2006) e apresenta o uso da Teoria das Situações Didáticas em Matemática em uma situação de ensino na escolarização obrigatória básica. Concomitantemente a essa prática, surgiu a Teoria da Etnomatemática de D’Ambrósio.

Brousseau (2006), além de identificar as características das Teorias das Situações Didáticas da Matemática, mostra seu entendimento sobre a Etnomatemática: "a Matemática que se manifesta em algumas atividades de cultura ou institucionais" (p. 438). Maranhão e Manrique (2014) mencionam que Brousseau (2016) propôs discussões sobre as duas teorias, promovendo reflexões sobre como esses conhecimentos são difundidos. Assim, as autoras acreditam que o artigo evidencia o conhecimento da prática, no qual o professor investiga suas práticas de acordo com o que lhe é apresentado nas duas teorias.

Esse artigo mostrou a Teoria das Situações Didáticas em Matemática sob uma nova perspectiva, utilizando para isso concepções docentes de Cochran-Smith e Lytle (1999).

Tinti e Manrique (2016) apresentaram um estudo intitulado “Teoria e prática na formação de professores que ensinam Matemática: que caminhos apontam experiências com o PIBID e OBEDUC", publicado no periódico Educação Matemática em Revista, da Sociedade Brasileira de Educação Matemática. O objetivo do estudo foi:

[...] analisar experiências vivenciadas na PUC-SP em dois programas de formação de professores vinculados à Capes, sendo o primeiro o PIBID - Programa Institucional de Bolsa de Iniciação à Docência - e o segundo, o OBEDUC - Programa Observatório da Educação, evidenciando caminhos para a Teoria e a Prática na formação de professores. (TINTI; MANRIQUE, 2016, p. 100).

Nesse estudo, os autores apresentam inicialmente os desafios e o contexto da formação de professores, referenciando os principais aspectos do Programa Institucional de Bolsa de Iniciação à Docência (Pibid) e do Programa Observatório da Educação (Obeduc).

Para a realização do estudo, Tinti e Manrique (2016) buscaram pesquisas desenvolvidas no Pibid e no Obeduc, selecionando duas dissertações do primeiro - Tinti (2012) e Correia (2012) - e uma dissertação do segundo - Silva (2012). A metassíntese qualitativa foi realizada buscando semelhanças, diferenças ou mesmo complementaridades entre os resultados das pesquisas.

Os estudos que investigaram o Pibid identificaram a importância de conhecer bem o contexto escolar; de ter acesso aos professores; da possibilidade do desenvolvimento de 
formações com eles; de experiências, projetos colaborativos e interdisciplinares; da parceria escola-universidade; da quebra do preconceito da escola pública, aumentando o interesse pela docência; da confecção e utilização de diferentes recursos metodológicos; da relação entre teoria e prática; da elaboração de artigos em escrita compartilhada.

Quanto ao Obeduc, Tinti e Manrique (2016) relatam que Silva (2015) identificou um espaço formativo colaborativo em diferentes momentos da carreira, espaço considerado uma comunidade de prática; existência de diálogo entre a pesquisa e a prática docente; e o favorecimento da parceria universidade-escola. Tinti e Manrique (2016) consideram ainda que ambos os programas, Pibid e Obeduc, indicam que a escola deve ser um lugar de aprendizagem para o docente.

Consideramos que esse artigo foi importante para a reflexão sobre as formações e práticas docentes e para mostrar como programas governamentais, como o Pibid e o Obeduc, podem influenciar e auxiliar essas formações.

Observamos que identificar como cada pesquisa realizou a metassíntese promove a reflexão sobre os possíveis caminhos metodológicos a serem seguidos.

\section{CONSIDERAÇÕES FINAIS}

A realização dessa modalidade de pesquisa, a metassíntese qualitativa, necessita que os dados apresentados nos resumos, capítulos e registros estejam claramente bem escritos e definidos para que sejam mais bem metassintetizados. Além disso, as investigações devem ser disponibilizadas na íntegra para a consulta e metassíntese dos dados. Tal disponibilidade na atualidade é mais acessível em face do favorecimento dessas pesquisas no banco de dados de Teses da Capes e em acervos digitais nos Programas de Pós-Graduação do País.

Os critérios de seleção das pesquisas devem ser rigorosos, sendo as investigações selecionadas por expressões-chave retiradas da temática e do objetivo do estudo, fazendo ainda filtros para se conseguir o número reduzido de pesquisas indicado por essa metodologia. Salientamos que a metassíntese qualitativa é uma das metodologias que demandam certo tempo para seu desenvolvimento e consolidação.

Acreditamos que este tipo de revisão sistemática auxilia áreas com temáticas que possuem grande número de investigações para que os pesquisadores possam refletir sobre os aspectos da área investigada. 


\section{REFERÊNCIAS}

ALENCAR, E. S. Formação de professores sobre o Campo Conceitual Multiplicativo: referenciais teóricos em pesquisas. 2016. p. 186. Tese (Doutorado em Educação Matemática) PUC-SP, São Paulo.

Conhecimento Profissional Docente de Professores do 5. ${ }^{\circ}$ ano em uma escola com bom desempenho em Matemática: o caso das estruturas multiplicativas. 2012. Dissertação (Mestrado em Educação Matemática) - UNIBAN, São Paulo.

BANCO de teses. Capes, 2017. Disponível em: <http://www.bancodeteses.capes.gov.br>. Acesso em: abr. 2017.

CAMPOS, E. G. J. D. As dificuldades na aprendizagem da divisão: análise da produção dos erros dos alunos do ensino fundamental e sua relação com o ensino praticado pelos professores. 2007. Dissertação (Mestrado em Educação) - Universidade Católica Dom Bosco, Campo Grande.

FIORENTINI, D. Investigação em Educação Matemática desde a perspectiva acadêmica e profissional: desafios e possibilidades de aproximação. ANAIS do XIII Conferência Interamericana de Educação Matemática, CIAEM, 2011.

Investigação em Educação Matemática desde a perspectiva acadêmica e profissional: desafios e possibilidades de aproximação. Cuadernos de Investigación y Formación en Educación Matemática, p. 61-82, 2013.

GARCIA SILVA, Angélica A. Fontoura. O desafio do desenvolvimento profissional docente: análise da formação continuada de um grupo de professores das séries iniciais do ensino fundamental, tendo como objeto de discussão o processo do ensino e aprendizagem de frações. 2007. Tese (Doutorado em Educação Matemática) - PUC-SP.

LAKATOS, E. M. Metodologia do trabalho científico: procedimentos básicos, pesquisa bibliográfica, projeto e relatório, publicações e trabalhos científicos. 7. ed. São Paulo: Atlas, 2011.

MARANHÃO, M. C. S. A.; MANRIQUE A. L. Pesquisas que articulam a Teoria das Situações Didáticas em Matemática com outras Teorias: concepções sobre aprendizagem do professor. Revista Perspectivas da Educação Matemática, v. 7, Número Temático, 2014.

MATHEUS, M. C.C. Metassíntese qualitativa: desenvolvimento e contribuições para a prática baseada em evidências. Acta Paul Enferm, p. 138, 2009.

MERLINI, V. L. As potencialidades de um processo formativo para a reflexão na e sobre a prática de uma professora das séries iniciais: um estudo de caso. 2012. Tese (Doutorado em Educação Matemática) - Pontifícia Universidade Católica de São Paulo, São Paulo.

OLIVEIRA, E. G. Raciocínio combinatório na resolução de problemas nos anos iniciais do ensino fundamental: um estudo com professores. 2014. Dissertação (Mestrado em Educação Matemática) - PUC-SP, São Paulo. 
ROCHA, C. A. A formação docente e o ensino de problemas combinatórios: diversos olhares, diferentes conhecimentos. 2011. Dissertação (Mestrado em Educação) - UFPE, Recife.

ROMANOWSKI, J. P.; ENS, R. T. As pesquisas denominadas do tipo estado da arte em Educação. Revista Diálogo Educacional, v. 6, n. 19, 2006.

SANTOS, A. D. O conceito de fração em seus diferentes significados: um estudo diagnóstico junto a professores que atuam no Ensino Fundamental. 2005. Dissertação (Mestrado em Educação Matemática) - PUC-SP, São Paulo.

Processo de formação colaborativa com foco no Campo Multiplicativo: um caminho possível com professoras polivalentes. 2012. Tese (Doutorado em Educação Matemática) PUC-SP, São Paulo.

SILVA JUNIOR, F. J. Intervenções didáticas no ensino de frações e a formação de professores. 2015. Dissertação (Mestrado em Educação Matemática) - UNIAN, São Paulo.

TINTI, D. S.; MANRIQUE, A. L. Teoria e prática na formação de professores que ensinam matemática: que caminhos apontam experiências com o PIBID e OBEDUC?. Educação Matemática em Revista, São Paulo, v. 49B, p. 98-106, 2016.

VOSGERAU; ROMANOWSKI. Estudos de revisão: implicações conceituais e metodológicas Revista Diálogo Educacional, Curitiba, v. 14, n. 41, p. 165-189, jan.-abr. 2014.

Data de recebimento: $29 / 05 / 2017$

Data de aceite: 25/11/2017 